\title{
P2P Object-based adaptivE Multimedia Streaming (POEMS)
}

\author{
Toufik Ahmed $\cdot$ Mubashar Mushtaq
}

Published online: 21 June 2007

(C) Springer Science+Business Media, LLC 2007

\begin{abstract}
Peer-to-peer ( $\mathrm{P} 2 \mathrm{P})$ paradigm has recently gained tremendous attraction and is widely used for content distribution and sharing. The future multimedia communication applications have to support the user's needs, the terminal capabilities, the content specification and the underlying networking technologies. They should be network-aware, topology-aware, and end-user-centric. Thus, in this paper, we use the characteristics of the object-based encoding scheme and P2P network topology to propose adaptive content delivery architecture for $\mathrm{P} 2 \mathrm{P}$ networks. We propose an efficient mechanism for transmission of real-time content over $\mathrm{P} 2 \mathrm{P}$ networks, called POEMS (P2P Object-based adaptivE Multimedia Streaming). This object-based audio-visual quality adaptive mechanism over P2P networks is mediaaware, network-aware, and user-centric that is carried-out through (1) selection of appropriate sending peers willing to participate in the streaming mechanism, (2) organization of sending peers by constructing an overlay network to facilitate content delivery and adaptation, (3) dynamicity management of peers when some peer enters or leaves the system to maintain an acceptable level of perceived video quality, and (4) ensuring the end-to-end QoS (Quality of Services) by orchestrating the overall streaming mechanism. The obtained results demonstrate that combining content adaptation using object-based encoding and advance network-aware peers selection based on peer monitoring leads to intelligent, efficient, and large-scale support of multimedia services over complex network architectures.
\end{abstract}

Keywords P2P streaming - Audiovisual content delivery - Quality adaptation · Quality of service (QoS)

T. Ahmed $(\bowtie) \cdot$ M. Mushtaq

CNRS LaBRI Lab., University of Bordeaux 1, 351, cours de la Libération, Talence Cedex 33405,

France

e-mail: tad@labri.fr

M. Mushtaq

e-mail: Mushtaq@1abri.fr 


\section{Introduction}

Multimedia communication is becoming an indispensable feature of networking environments. Audio and video content are becoming more and more popular on the Internet. Many systems are being designed to carry this media such as video conferencing, video on demand, IP Telephony, and Internet TV. In addition, peerto-peer (P2P) networks are radically changing the way people consume and interact with contents. People are using P2P networks for sharing their documents, photos, music, movies, and much more. Multimedia (Audio/Video) data transfer over P2P networks has a major influence on Internet traffic. In our study, we are more concerned with the transfer of media content between peers. Media transfer can be done in two ways: downloading or streaming. In the downloading mode, the entire contents are transferred/copied to another system after which the receiver can open the received file. In the streaming mode, some small parts/segments of the file are transferred and played back immediately. These smaller segments are transferred according to the actual end-to-end data rate transfer. Being real-time application, streaming mode is more challenging and still needs deep investigations.

Audiovisual applications have adaptive bandwidth capabilities, but have stringent delay, jitter, and packet loss requirements, which are not supported by current IP networks. It is therefore essential to design multimedia applications capable of adapting to system and network resource constraints, (i.e., networkaware) whilst ensuring that end-user requirements are taken into consideration (user-centric). Figure 1 shows framework for advance content adaptation by integrating the content, the user, the terminal, and the network characteristics. This content adaptation is subject to many requirements. Media characteristics are the key element for the optimal adaptive streaming mechanism. Descriptive information related to user characteristics (e.g., user information and preferences, usage history, presentation preference, audio language preferences, subtitles language preferences), terminal capabilities (e.g., codec capabilities, display capabilities, adaptation capability), network characteristics (e.g., network capabilities and network conditions), and natural environment characteristics (e.g., location and time), are essential for end-to-end QoS provisioning over heterogeneous environments.
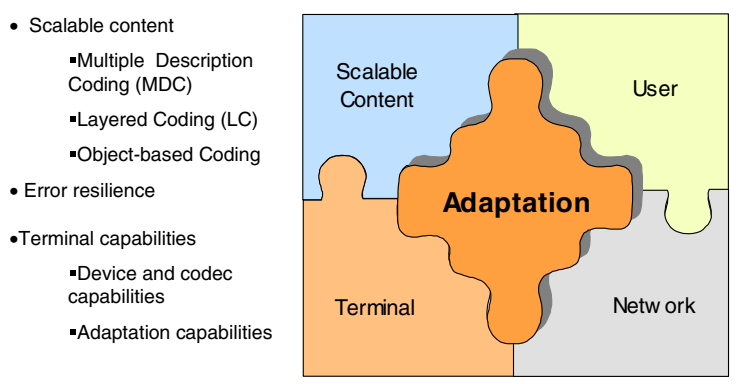

-User characteristics

-User Information and Preferences

-Usage history

-Presentation Preferences

-Network characteristics

-Supported QoS mechanisms (DiffServ, resource reservation, MPLS)

-Network capabilities (static)

-Network conditions (dynamic)

Fig. 1 Overall audiovisual content adaptation by integrating content, terminal, user and network 
However, traditional network infrastructures are not able to incorporate this new dimension of real time traffic in their existing characteristics. In fact, it is very hard to meet the above requirements over the Internet in the presence of huge volumes of noisy traffic, heterogeneous receiver capabilities, and a multitude of content coding techniques. Furthermore, a single sender is unable to fulfill requirements for streaming a huge media file itself. A lot of users are connecting to Internet using ADSL (Asymmetric Digital Subscriber Line) or cable modems. A typical ADSL or cable modem installation offers three to eight times more bandwidth for downlink than uplink. Most of the users are clients who are surfing the Internet to download only and they don't use uplink to publish any data. The difference of uplink and downlink capacities makes it impossible to get whole media contents fully from a single user. Subsequently, it is essential to select multiple senders to get complete contents. Receiver can attain required bandwidth by combining the uplink bandwidth of all senders. This objective is realized in P2P networks. Participating peers in $\mathrm{P} 2 \mathrm{P}$ network collaborate with each other to accomplish tasks/objectives. In the $\mathrm{P} 2 \mathrm{P}$ model many peers/nodes subscribe to the system. Some of these having some contents to share with other peers and some subscribe to search some contents. In particular, the characteristics of $\mathrm{P} 2 \mathrm{P}$ networks make it convenient for media streaming applications by sharing the available resources of different peers. However, P2P architecture is dynamic in nature. It connects a large number of peers that may reduce their bandwidth sharing capabilities. Some peers can enter or leave the P2P system without any prior notification. The dynamicity of P2P brings up some problems which can affect and reduce the overall quality for streaming applications. Thus, a careful analysis is required to manage all the streaming mechanism over P2P platforms.

In this paper, we propose an object-based adaptive streaming mechanism over $\mathrm{P} 2 \mathrm{P}$ networks. Our proposed quality adaptive streaming mechanism is media aware, network-aware, and user centric. Media-awareness is taken into consideration for peer organization. Important parts of the video scene (i.e., an important audiovisual object) are delivered from best peers (i.e., peer providing the best (QoS) quality of service). Network-awareness is taken into consideration by peer monitoring to select the appropriate set of peers among many available peers. User-centricity allows the receiver to coordinate the overall streaming mechanism based on its capabilities and requirements. The receiver's main objective is to maximize the received throughput (total number of received packets/received audiovisual objects) whilst minimizing the packet loss ratio to ensure improvement in the overall QoS.

The rest of this paper is organized in different section as follows. A content adaptation technique for media streaming state of the art is presented in section "Content adaptation for media streaming". It gives an overall overview of architectures, techniques, and methods used for media streaming adaptation. Section "Multimedia streaming over P2P networks-state of art" presents the proposed object-based audio-visual quality adaptation for P2P network. Section "Objectbased audio-visual quality adaptation for P2P Networks" presents some performance evaluation. Concluding remarks are made in section "Performance evaluation',. 


\section{Content adaptation for media streaming}

The end-to-end QoS delivery chain should support heterogeneity in networks and terminals capabilities. Thus, adaptation techniques should be used to cope with these issues. Content adaptation can be done at the sender, at the receiver, or within the network using a specific adaptation engine. Content adaptation is essential to deal with channel bandwidth variation and heterogeneity at many levels. This section summarizes the main techniques used to provide a seamless multimedia delivery and adaptation.

\section{Simultaneous store and stream}

"Simultaneous Store and Stream" also know as Simulstore [1] is the technique used to store at the server different streams with different spatial resolutions, temporal resolutions and SNR levels. The client connects to the server and selects the appropriate stream from a multitude of stored streams. The quality of the video is not degraded, because each stream is coded optimally. Furthermore, this technique has an easy selection of the appropriate stream at server side and low complexity at server and client.

Simulstore can easily be combined with end-to-end retransmission but it has a major disadvantage that streams cannot cope with degradations in network conditions, hence, cannot be adapted to network conditions.

\section{Stream switching}

Streams switching overcomes the disadvantage of Simultaneous Store and Stream by adapting the video transmission rate to network conditions. This technique is developed specially for controlling Simulcast streaming. It works as follows. If the transmission condition changes (less or more network capacity), another stream is selected. This can lead to a synchronization problem. To overcome this problem, streams are synchronized according to intra-coded frames (I-Frame) or special switching frame (SP-Frame). Signaling in this case is crucial for stream switching.

In [2] and [3], authors propose a seamless switching scheme for scalable video bitstreams. In this proposal, small bandwidth fluctuations are accommodated by the scalability of the bitstreams, while large bandwidth fluctuations are tolerated by switching between scalable bitstreams.

In [4] stream switching is also investigated in case of unicast and multicast video streaming. The proposed system can provide network adaptation with receiverdriven congestion control by estimating the network conditions. Clients are able to adjust their subscription levels by joining or leaving multicast groups according to their estimated bandwidth. This allows users to switch up and down over differentrate bitstreams with minimized drift errors. Each group delivers a particular quality of video stream.

RealVideo products developed by real network employs the method of stream switching called SureStream [5]. It consists of multiple streams encoding at different rates and stored in a single file. It provides a mechanism for servers and 
clients to detect changes in bandwidth and choose an appropriate stream. As a user is watching a video, the delivery bit rate can shift up or downshift, adjusting to changing network bandwidth conditions, to ensure the user a smooth playback experience. SureStream works for both live and on-demand content.

\section{Simulcast}

Simulcast is simultaneous transmission of different streams with different spatial resolutions, temporal resolutions and SNR level in multicast network. The client selects the appropriate stream out of multitude of streams. It can switch from one stream to another very easily. The client is a simple single layer decoder able to up and down over different-rate bitstreams with minimized drift errors. Each multicast group delivers a particular quality of video stream.

\section{Real-time encoding}

Real-time encoding is the process of encoding the video stream right before the transmission process. The client connects to the servers and specifies the encoding parameters and its requirements. The server processes to encode the video in realtime to handle user needs. This technique results in a high complexity at the server side.

\section{Content adaptation engine}

Content adaptation can be performed in the core network or in the access network using specific application level gateway called Content Adaptation Engine (CAE). In general, CAE uses scalability features of multimedia contents. Some techniques such as transcoding, transrating, and color/pixel downsampling are in common use. Transcoding aims to transform from one media format to another media format. For example transcoding from MPEG-2 format to H.264 format. This allows bit rate reduction, frame rate reduction, and temporal and special downsampling [6]. The transcoding is applied by decoding the video to raw format (uncompressed video) and then re-encoding the video to the target format. There are mainly two drawbacks when using this approach. First, the quality of the result format is generally lower than the original format. Second, media transcoding generally requires extensive computation and large storage spaces, which makes this approach very expensive. The advantage in transcoding is that it allows media adaptation both at the server and in the network. However, it can be efficient using a dedicated media gateway $[7,8]$.

\section{Media caching}

Multimedia caching is an important technique for enhancing the performance of streaming system. Proxy caching has been the key factor in the scalability of the Web, because it reduces the network load and access latency. Video caching aims to store some part of the video near the clients. It allows improving the quality of the 
delivered stream when there is a presence of bottleneck links between the server and the client. Many studies have experienced the media proxy caching [9-14].

\section{Video smoothing}

The rate of the encoded video stream is in general a variable bit rate having a bursty behavior. The bursty nature of this traffic causes in the majority of case network congestion. In order to reduce the variability of the traffic and bandwidth requirement of the video stream, smoothing techniques are necessary. Smoothing makes use of a client side buffer to receive data in advance of playback [15-19].

\section{Swarming multimedia}

Swarming is a technique used for content distribution over large scale networks [20]. In swarming a huge file is divided into many small pieces for distribution. Clients request different pieces from the server or from other clients. Each client becomes server for those pieces of file which it has downloaded. When all pieces are downloaded, clients can re-construct the whole file. Bit Torrent systems are based on the philosophy of swarming and this approach can be enhanced for multimedia streaming over P2P networks. IceShare is another pseudo-P2P system that relies on a traditional client-server model for managing transfers between IceShare peers on the network.

\section{Multimedia streaming over P2P networks-state of art}

Peer-to-peer architecture has gained large attention recently, especially in the era of multimedia streaming. The wide spread of P2P is expected to re-shape the Internet community in the near future. A lot of work is going on for streaming over P2P. In this section, we have classified some of the works being done in the same area.

Reza et al. have proposed a framework PALS [21] for P2P adaptive layered streaming. 'PALS' is a receiver centric framework, where a receiver coordinates delivery of a layer encoded stream from multiple senders. A peer selection criterion has been proposed to maximize the overall throughput. In this framework initial peers are selected on a random basis because there is no information available at the start of the streaming mechanism. Peer selection is an iterative process. Each time a new peer is admitted and if it enhances the overall throughput it is kept as sender peer otherwise it is dropped. For quality adaptation, the receiver manages its buffer regularly on the basis of packets consumption and sends the buffer state to each sender. The QA mechanism for PALS determines inter-layer bandwidth allocation for a period of time rather than on a per-packet basis.

Hefeeda et al. [22] proposed a mechanism 'PROMISE' for P2P media streaming using CollectCast. They proposed an idea for collaborating with multiple sender peers for media streaming. A comparison is done for different selection techniques, i.e., "Topology Aware Selection", and "End-to-End 
Selection'. In the Topology Aware selection technique all the shared communication paths/links are considered for best selection of the sending peers while in the end-to-end selection technique these shared segments/links are not considered during selection of the sender peers. To ensure constant streaming, status of all the sender peers are monitored regularly. The monitoring is performed by collecting the statistics on the loss rate and streaming rate contributed from the each peer. Dynamic switching is performed in the case of peer failure and network fluctuations. In the case of peer failure, new best peer is selected while in the case of network fluctuations new rates are assigned for the active peers or by adding or removing the sender peers from the active set of peers. In contrast to the 'PALS' and 'PROMISE', we proposed a different mechanism in 'POEMS' for the selection of sender peers and their efficient switching. In 'POEMS' peers selection is performed on the basis of round trip time 'RTT' measurement among for each receiver and each sender peer. An active monitoring is performed for the continuous measurement of 'RTT' to switch to the new best available sender peer. The proposed selection criterion is helpful to select best sender peers right from the beginning of the streaming mechanism.

In [23] a distributed mechanism is described for video streaming from multiple senders. The streaming mechanism is based on a rate allocation algorithm (RAA) and a packet partition algorithm (PPA). The 'RAA' runs at the receiver end to determine the sending rate based on the available bandwidth. The 'PPA' runs at the senders' side to minimize the startup delay of packets. PPA ensures that each packet is sent by only one sender. In our proposed mechanism 'POEMS', receiver peer selects different sender peers to receive different video objects of the media file. Furthermore, more than one sender peer is selected for one media object, while each object consisted of multiple layers.

Yeo et al. [24] have proposed a framework for the distribution of multicast video streaming across heterogeneous networks. A tree based overlay network is constructed and Round Trip Time (RTT) is used for search criterion. They arranged all the clients in four groups based on RTT values. The overlay trees are constructed similar to Prim's algorithm which is commonly used to derive the minimum spanning tree in multicast routing. This RTT based tree overlay construction is proposed for the improvement of overall QoS. In 'POEMS', we used 'RTT' measurements for the best sender peers selection and the organization of peers in different overlay networks.

Loeser et al. [25, 26] have proposed architecture of an intelligent QoS aware P2P multimedia network. The proposed architecture is based on the direct RSVP enabled point to point streaming connections between any two peers. They treated the Active Rendezvous Server (ARS) not only as directory server (lookup services) but also for contents distribution with in the networks. The ARS also determines the most efficient peer by considering the workload of source peer and workload at each network link. Generally, this architecture has been proposed for autonomous networks e.g., universities, hotels, large companies, and institutes etc.

In [27], an architecture 'P2CAST' is presented for the VoD Services that uses the peer-to-peer approach and is based on patching techniques. The architecture relies on only unicast connections among the peers. The key idea behind the architecture 
is that each client behaves as server for the other client and provides some patches of data to other clients. This approach is used to overcome the overload on a single media server. The main theme of this approach is to scale better than traditional client server architecture by constructing the application overlay and to provide continuous playback.

Tran et al. [28, 29] have presented an architecture for media streaming called 'ZIGZAG'. They proposed a method for clustering the peers into a hierarchy for their management and organization. Zigzag uses a multicast tree construction and maintenance approach based on a hierarchy of bounded-size clusters that helps in reducing the number of processing hops to avoid the network bottleneck.

'CoopNet' [30] is a solution for distributing streaming media content using cooperative networking. The main objective of CoopNet is to distribute media contents to a large number of hosts in a scalable way. The work is in the context of a client-server model to overcome the problem arising when the server is overwhelmed. In the proposed solution, all clients cooperate with each other for the distribution of data. Authors used Multiple Description Coding (MDC) for data encoding so that original content can be regained in the case of multiple failures. In [31], 'CoopNet' approach is applied for supporting resilient live streaming using application-layer multicast over an unreliable set of peers. The resilience is provided by introducing redundancy both in network paths and data.

In our proposed streaming mechanism 'POEMS', we tried to acquire the benefits of MPEG4 part 2-object-based coding scheme while applying a P2P environment. The organization of the peers in different overlay networks on the basis of end-to-end 'RTT' measurement and offered video quality makes it convenient to manage the dynamicity of the peers, and thus improve the overall QoS. Moreover, smooth delivery of the most important objects are ensured even in the worst scenarios.

\section{Object-based audio-visual quality adaptation for P2P networks}

\section{Object-based coding}

The Object-based Encoding was introduced in MPEG-4 part 2 standard. This coding technique allows the encoding of different audio-visual objects (AVOs) in the scene independent of each other.

Lets take an example of an MPEG-4 scene which consists of one or more AVOs, each of which is characterized by temporal and spatial information and may be encoded by a scalable (multi-layer) or non scalable (single layer) encoding scheme. One layer is composed of a sequence of a Group of Video-Object-Plane (GOV). A Video Object Plane (VOP) is similar to the MPEG-2 frame. A VOP supports intra coded (I-VOP), temporally predicted (P-VOP), and bi directionally predicted (BVOP) encoding. The hierarchical composition of an MPEG-4 scene is depicted in Fig. 2.

To take benefits from the object-based encoding, we propose to use an intelligent adaptation to cope with network congestion and client terminal 


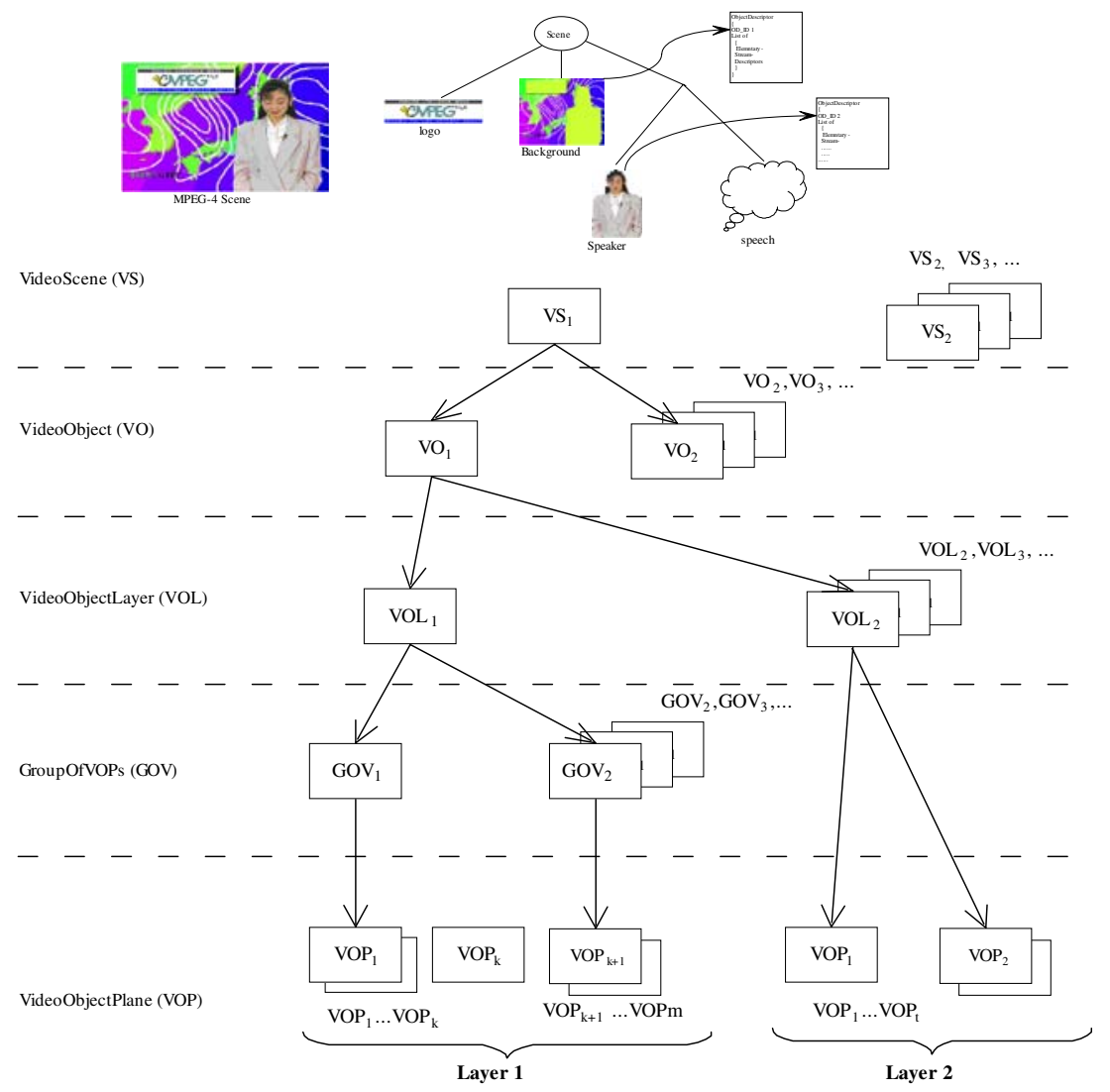

Fig. 2 Hierarchical composition of an MPEG-4 scene

heterogeneity over P2P network. A mechanism is proposed in [32] to classify AVOs at the video server. AVOs classification allows affecting a relative priority score (RPS) to each object. Based on RPS, an adaptive application level framing protocol with unequal forward error protection and fine grained video rate adaptation was proposed. Several methods can be used for AVOs classification. During scene creation, authoring tools can be used to affect the adequate priorities to each object in the scene. For scenes with no assigned object priorities, related metadata such as MPEG-4 object descriptors, MPEG-7, and MPEG-21 metadata can provide the relevant information needed to handle object priority and affecting the RPS value to each AVO. P2P streaming mechanism has to distinguish between important AVOs and less important one for providing better quality with efficient adaptation. 
We proposed a quality adaptive mechanism for the packet video streaming over $\mathrm{P} 2 \mathrm{P}$ networks based on object-based coding 'POEMS'. In the object-based adaptation, video scene is classified according to the priority of different objects. The main objective behind this mechanism is to ensure smooth play-back for the most important object. Our proposed streaming mechanism is based on the selection of best sender peers from the set of peers (potential peers) who have required contents and they are ready to participate in streaming mechanism. All the potential peers are organized in an overlay network, which is build on the top of P2P network. We classify the potential peers in the overlay network according to their offered video quality and their QoS capabilities (RTT values). Active monitoring is performed to evaluate current network condition (i.e., if any sender peer goes down or any of the network links is going to be congested due to background traffic). In the above stated problem, peer switching is performed by selecting the new best peer to ensure smooth media streaming. An efficient peer selection mechanism is described in the next subsection.

\section{Efficient peer selection for object-based adaptive streaming in 'POEMS'}

We discussed earlier that video packets are encoded using object-based encoding scheme. Each object is assigned a certain priority score. The receiver peer receives different media objects from different sending peers. Most important video objects are delivered from the best senders peers. Upon receiving audiovisual objects, the receiver reconstructs the original video stream and starts rendering the MPEG-4 scene for play-back.

Let there be ' $n$ ' numbers of sending peers (potential peers) present in the network. Each peer may serve one or more audiovisual objects. Providing QoS of each peer is estimated with active monitoring using end-to-end RTT. This RTT measurement is performed between each sender peer and receiver peer. We classified all the sender peers on the basis of their offered Audio Video Objects (AVOs) and provided QoS, i.e., estimated RTT between sender and receiver peer, and multiple overlay networks are constructed for each audiovisual object. All peers with the same AVO are organized in the same virtual overlay network. With in the same overlay network, all peers are organized on the basis of provided QoS, i.e., RTT estimates. The sender peers with the lowest RTT values are known as the best sender peers and provide the best QoS. A pointer is set to the peers providing the best quality in each overlay network as shown in Fig. 3. The receiver selects the best sender peer from each overlay networks to start streaming. It is quite possible that there are senders having different media objects and present in more than one overlay networks. In our proposed mechanism peers containing important audiovisuals objects are assigned highest priority than peers offering less important audiovisual objects. Once a peer is selected for a high quality object then it is marked as unavailable in all other overlay networks to avoid its selection for low quality object. It is helpful to avoid congestion over certain network links which results in improvement of overall streaming quality. 


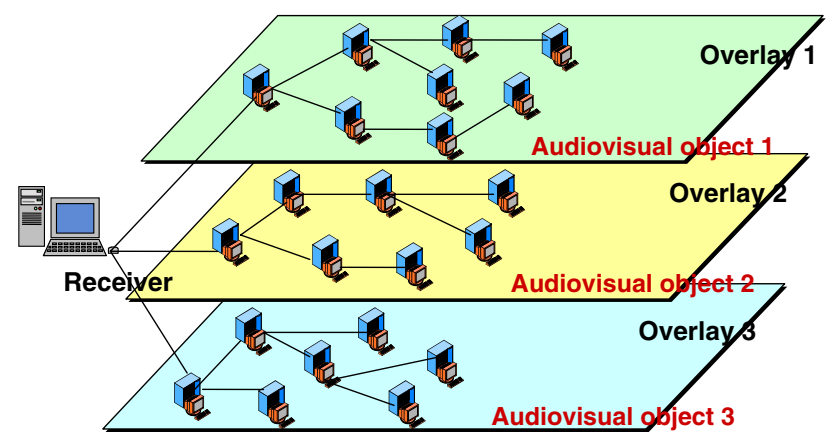

Fig. 3 Example of overlay networks construction

At the start of streaming, the receiver peer receives video packets from different senders and a certain amount of these video packets are stored in a buffer before actual playback. The size of the buffer depends on actual playback rate and on service configuration. For example, in VoD, 5-10 sec are sufficient for buffering the received data. For our proposed mechanism, we attached independent buffers at the receiver end for buffering all received audiovisual objects. A certain threshold value is set for each buffer which is determined by RTT estimates between sender and receiver peer and other transmission delay factor etc. The threshold value is proposed to be $50 \%$ of the buffer size. The actual buffer size and threshold value depends on the video play rate and current network conditions. If at any moment in time the received packets in the receiver side buffer are less than the threshold value, the receiver must select another sending peer for the same video quality. The peer switching mechanism is described in next section.

Peer switching mechanism

$\mathrm{P} 2 \mathrm{P}$ architecture is dynamic in nature, where peers can enter or leave the system without any prior notification. Thus, dynamicity management becomes more crucial to avoid the risks occurred, especially when any active sender peer leaves the system. For the better provision of the QoS and smooth contents delivery, streaming continuous monitoring is used to maintain the peers in the overlay network. In the receiver centric systems, receiver peer orchestrates the whole streaming mechanism and to avoid said problem receiver peer perform active monitoring of the sender peers. If a sender peer goes down, the receiver peer can select the next sender peer from the overlay network by readjusting the pointer in the overlay networks. In the proposed system, we arranged all the sender peers in the virtual overlay networks in the form of a ring. In Chord [33], peers are arranged in ring on the basis of hash values to facilitate lookup services in scalable distributed system. In our proposed solution, we organized the peers on the basis of offered AVO and RTT values. In this formation, we need to readjust the two links in the case of arrival or removal of any peer in the system. It enhances the scalability for the streaming mechanism. The receiver peer keeps track of the peers entering or leaving the overlay networks, whenever a peer offering better QoS enters the system or any of the active peers 
leave the system, peer switching is performed. In the flash crowd some peers may enter or leave the system just for a short period of time, i.e., not in stable condition. In this case, it is not recommended to perform peer switching each time because peer switching also incurs some overhead. To avoid such oscillation effects while updating peers at each RTT calculation, we applied a smoothing technique which is based on Exponential Weighted Moving Average (EWMA) [34]. It is an Exponential Smoothing technique that employs one exponential smoothing parameter to give more weight to recent observations and less weight to older observations and vice-versa as presented in the following equation:

$$
X \leftarrow(1-\lambda) * \mathrm{RTT}+\lambda * X
$$

When choosing " $\lambda$ ", it is recommended to use small values (such as 0.2 ) to detect small shifts and larger values (between 0.2 and 0.4 ) for larger shifts. In our case we set lambda $=0.2$ to detect the small shifts for the simulations.

\section{Performance evaluation}

Network architecture

We used the virtual network topology shown in Fig. 4 to simulate the object-based video streaming mechanism over P2P networks. We have distributed media contents

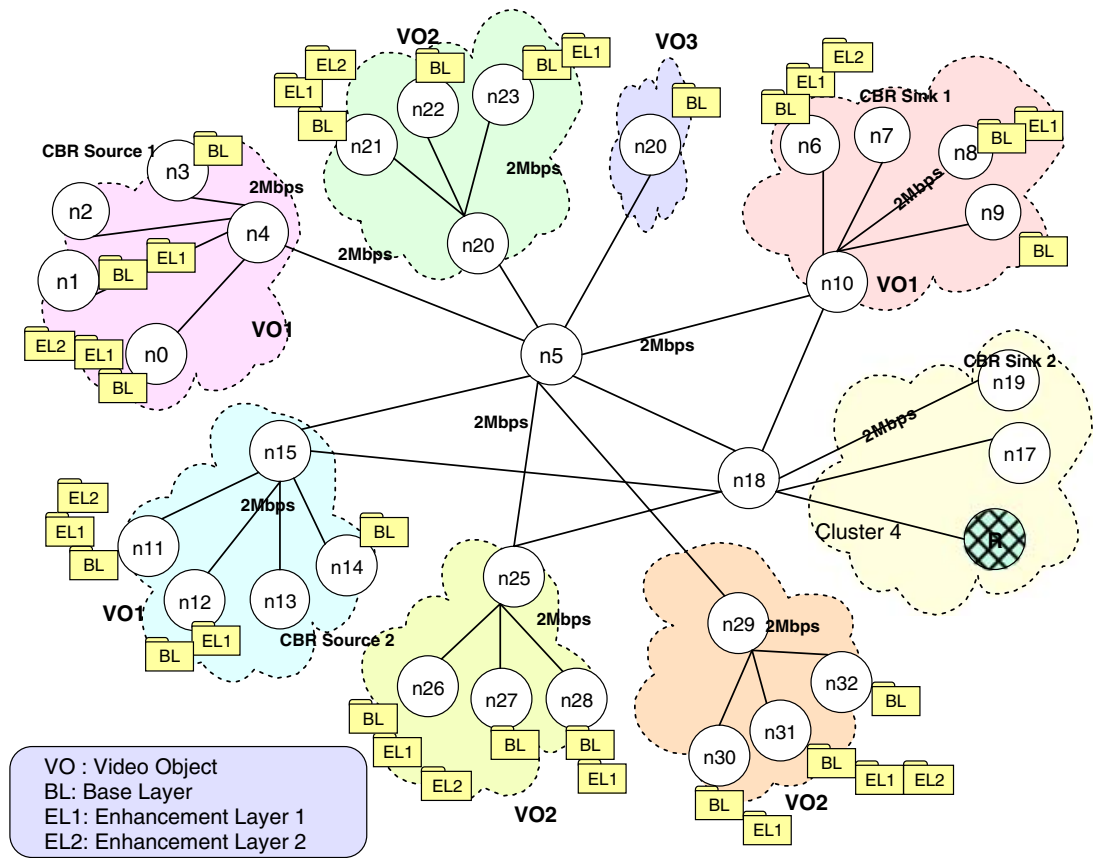

Fig. 4 Network topology 
to different sender peers participating in the streaming mechanism. Our proposed mechanism is receiver centric in the sense that the receiver is in charge of selecting the best sending peers. Receiver peer ' $R$ ' tracks ' $R T T$ ', among each sending peer and itself. This "RTT" monitoring is used to construct different overlay networks as explained in section "Efficient peer selection for object-based adaptive streaming in 'POEMS','. The bandwidth for all the network links is $2 \mathrm{Mbit} / \mathrm{s}$. Thus, it is impossible to get the whole content from a single sender.

\section{Audiovisual objects traffic model}

The audiovisual objects traffic is obtained from the MPEG-4 trace file [35]. In our simulations, we simulated the MPEG-4 ISO Akiyo scene the MPEG-4 presentation, which is composed of three visual objects for video and one object for audio: AO (audio speech), VO1 (background), VO2 (speaker) and VO3 (logo). A relative priority score to each object as follows:

- AO has the priority 1. It is the most important object in this scene. Simulation results of this object are not shown since we are more concerning with video objects adaptation.

- VO1 and VO2 have the priority 2.

- VO3 has the priority 3. It is the least important object in the scene.

Figure 5 shows the bit-rates of the MPEG-4 video objects to be sent from the sender peers to the receiver peer during a period of $60 \mathrm{sec}$. The complete scene along which is composed of three video objects is shown in Fig. 5(a). The Audio Object is a constant bit rate of $172 \mathrm{Kbits} / \mathrm{s}$. VO1 and VO2 are composed of three Layers: Base Layer, EL1 (Enhancement Layer 1) and EL2 (Enhancement Layer 2). In the encoding of the video, each object can be encoded into one base layers and many enhancement layers, but for the simplicity we divide each video object in one base layer and two upper enhancements layers which are decodable to its lower layers. Furthermore, for each independent video object (layer), we choose different sender peer. The purpose to choose different peers was to see the behavior of system when a lot of peers are coordinating with each other. The throughput for VO1 and VO2 is shown in Figs. 5(b) and (c), respectively. VO3 is composed of only one layer. The throughput of VO3 is shown in Fig. 5(d). The peak and average streams rate used for simulations are presented in Table 1.

We performed simulations to validate the audiovisual object-based quality adaptation mechanism over P2P network. We ran the simulations for two scenarios. We used two CBR sources for the background traffic to overcharge network links.

Scenario 1: Simulation with quality adaptation mechanism is performed by sender peers selection and their switching based on end-to-end active monitoring. If any node is going to be congested or it is down an alternative peer is selected to ensure reception of video objects according to their respective priority.

Scenario 2: Simulation without applying any quality adaptation mechanism. The sender peers are selected randomly. There is no peer switching performed even if 
(a)

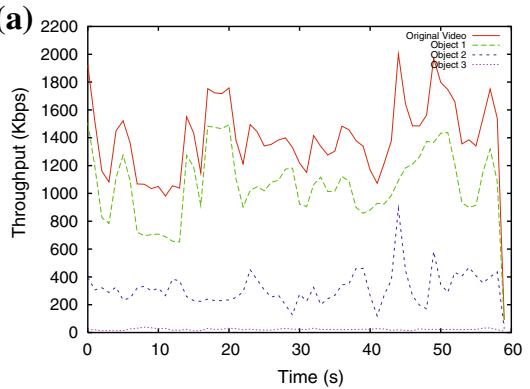

(c)

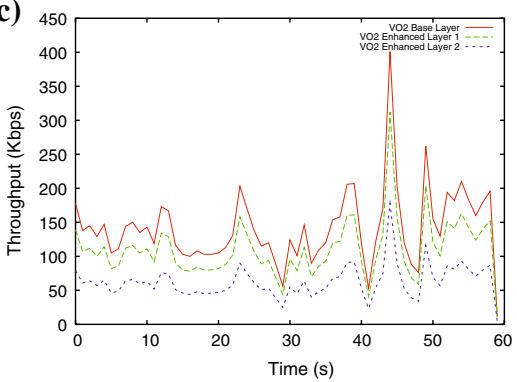

(b)

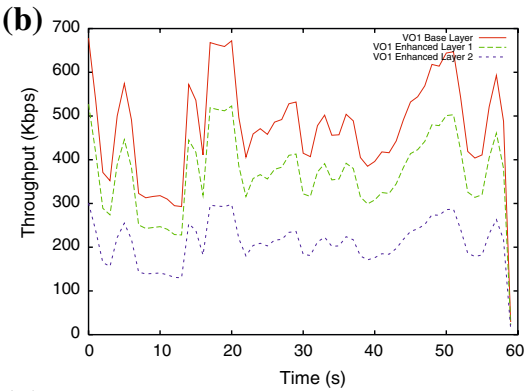

(d)

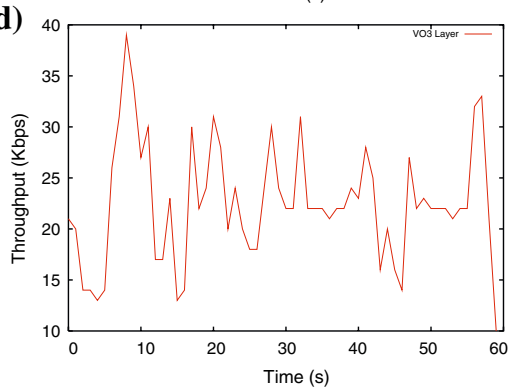

Fig. 5 (a) Original video; (b) Video object 1; (c) Video object 2; (d) Video object 3

Table 1 Average and peak rate for each layer

\begin{tabular}{lllllllllll}
\hline & VO1 & VO1 BL & VO1 El1 & VO1 E12 & VO2 & VO2 BL & VO2 El1 & VO2 E12 & VO3 \\
\hline Peak rate (Kbit/s) & 1507 & 678 & 527 & 301 & 893 & 401 & 312 & 178 & 39 \\
Average rate (Kbit/s) & 1053 & 474 & 368 & 211 & 315 & 142 & 110 & 63 & 22.50 \\
\hline
\end{tabular}

a particular peer becomes very congested because of background CBR traffic or if any of the sender peers are down during transmission of video packets.

Figure 6(a) shows the received throughput at receiver node " $R$ ', for each scenario i.e. with our proposed quality adaptation streaming mechanism (scenario 1) and without applying quality adaptation mechanism (scenario 2) along with the expected video layers quality when using the three different quality layers. We observed that adaptation mechanism maximizes the received throughput compared to the scenario without quality adaptation. Even with quality adaptation mechanism the received throughput is less than the expected one because of the heavy stress of the network created by CBR/UDP traffic. The background CBR traffic results into a lot of packet drops which is presented in Fig. 6(b). Packet drop ratio is much less in the scenario 

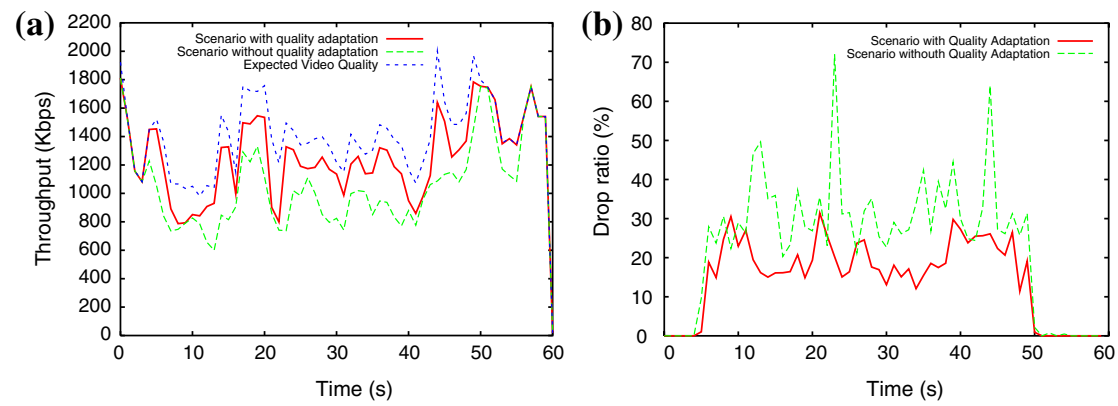

Fig. 6 (a) Received throughput; (b) Packet drop ratio

with quality adaptation compared to scenario without adaptation. This enhanced throughput and lower packet drops result in a significant improvement of the overall QoS.

Figure 7(a)-(c), describes the expected and received throughput for Base Layer, Enhancement Layer 1 and Enhancement Layer 2 for VO1. We can observe that there are some packet drops for EL1 and EL2. So, reception of Base Layer (which is more important) can provide smooth quality for VO1 in the scene for at-least most important object. Figure 8(a)-(c), represent the received and expected layers for $\mathrm{VO} 2$. We can see that there are many packet drops in the case of El1 and El2, while reception of the Base Layer is smooth.

Received and expected throughput for VO3 is shown in Fig. 9 and audio stream/ object is presented in Fig. 10. VO3 consists of only a single Base layer and it has the least priority, so a lot of packet drops for this video object is not hazardous since it was streamed from lower priority peers. Figure 11(a) and (b) represent packets drop for each layer of VO1, VO2 and VO3 for scenario 1 and scenario 2, respectively. As presented in this Fig. 11(a), the important layer (i.e., Base Layer) of important audiovisual objects experiences lower packet drops in the scenario using our adaptation mechanism (scenario 1) compared to the scenario without adaptation (scenario 2). Here, we noticed that packet loss to base layer is too small so use of Forward Error Correction (FEC) is not required for base layer, use of FEC will simply add additional overhead. In this paper we are not dealing the error correction techniques, rather we aim to enhance the overall QoS by smooth delivery of most important objects (Base Layers).

We combined all the video objects to construct the original video scene based on the video quality received during the simulation time for both scenarios. These videos are used to evaluate the PSNR and SSIM measurements. Peak signal-to-noise ratio (PSNR) is commonly used for measuring picture quality degradation. The Structural SIMilarity (SSIM) index is a novel method for measuring the similarity between two images. The comparison results for PSNR and SSIM of the videos generated in both scenarios to the original video are shown in Figs. 12 and 13, respectively. PSNR and SSIM measurements shows a noticeable improvement in the received video quality when our proposed quality adaptive streaming mechanism is applied. Figure 14(a) and (b) represent the effects of PSNR ratio 
Fig. 7 (a) Received base layer for video object 1; (b) Received enh. layer 1 for video object 1; (c) Received enh. layer 2 for video object 1
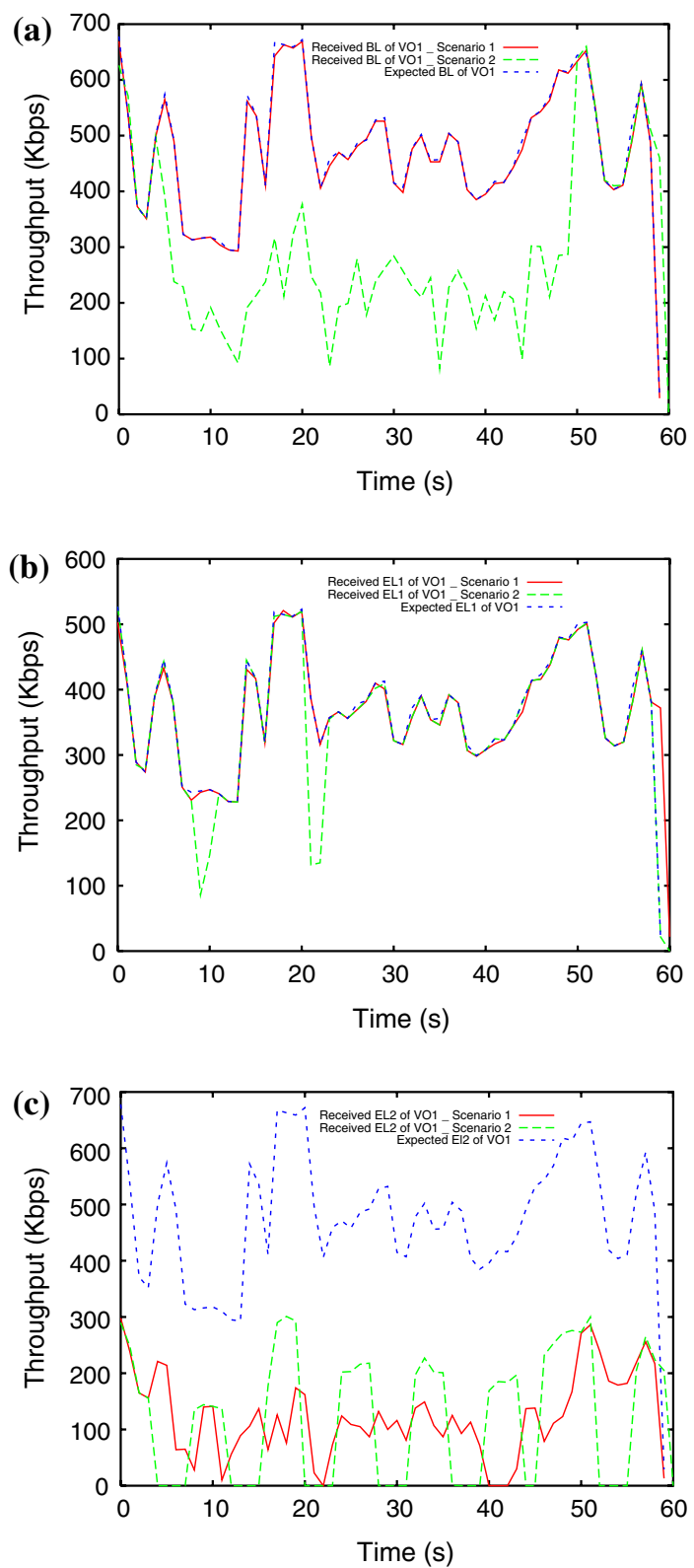

on the snapshot for AKIYO video sequence. For the logo object, our team logo was used with the original AKIYO video sequence. Here, in this paper we show only one video frame sequence number 201 to show the difference in received video quality in both scenarios. 

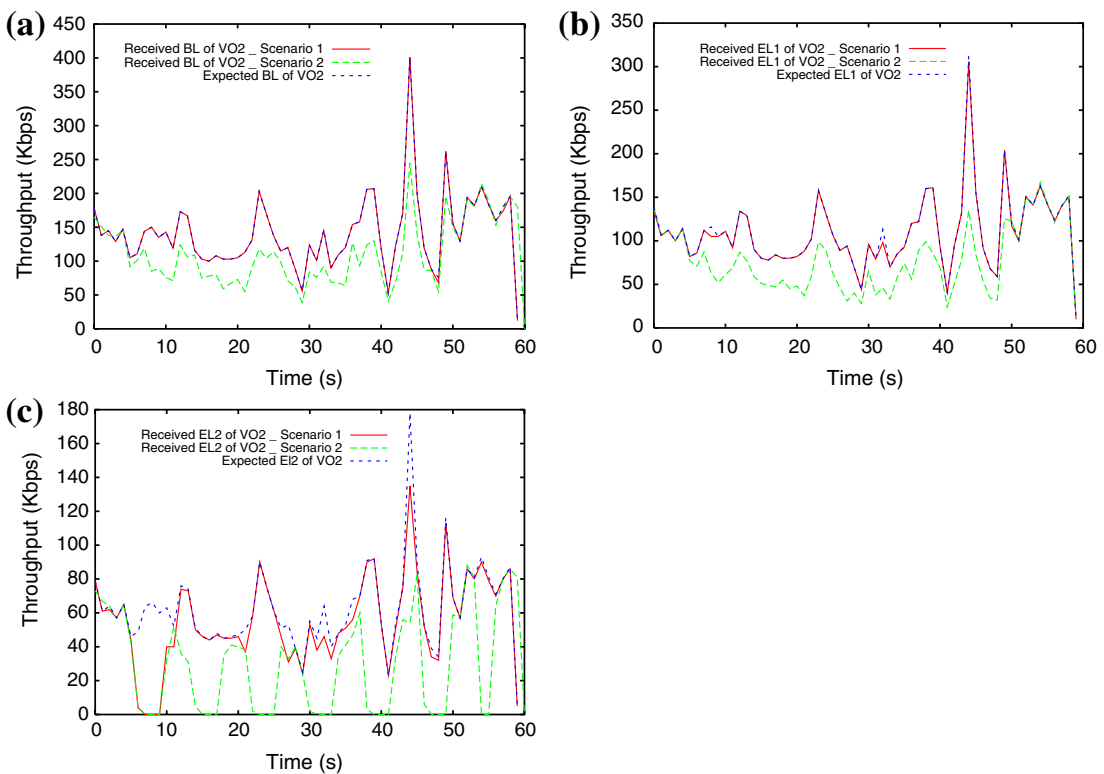

Fig. 8 (a) Received base layer for video object 2; (b) Received enh. layer 1 for video object 2; (c) Received enh. layer 2 for video object 2

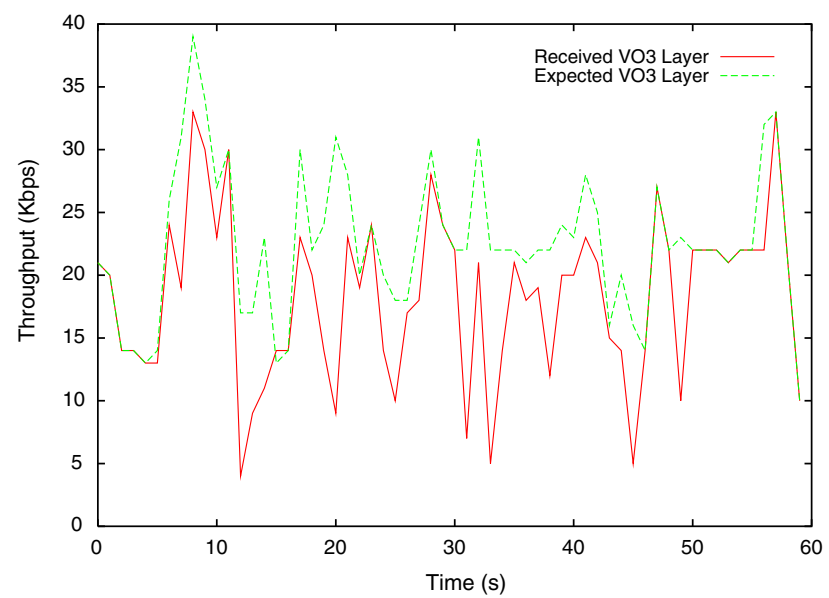

Fig. 9 Received and expected throughput for VO3

\section{Conclusion}

In this study, we investigated audiovisual object-based quality adaptation mechanism over P2P networks from multiple senders to a single receiver. We proposed a dynamicity controlled mechanism to offer better QoS (Quality of Service) to the 


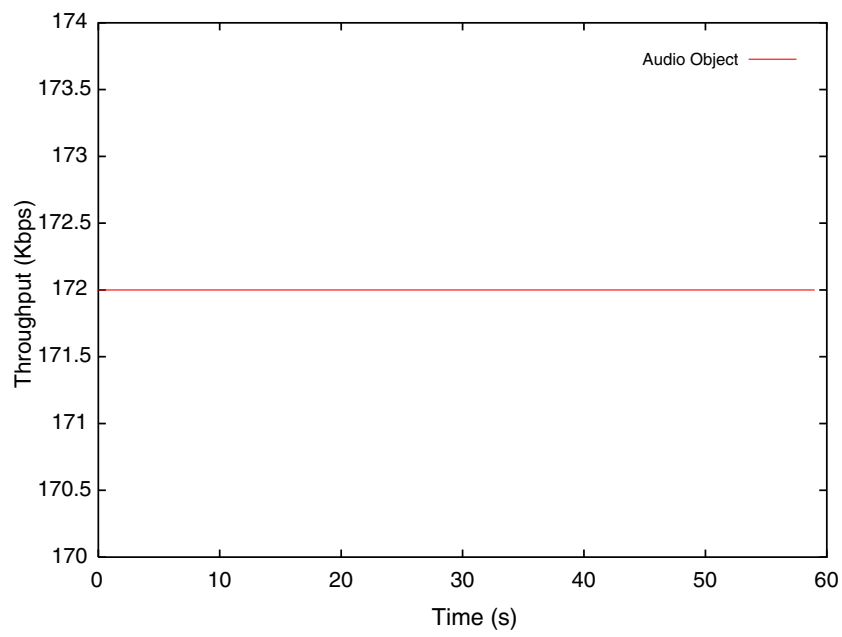

Fig. 10 Audio stream

Fig. 11 (a) Packets drops for each layer in scenario 1; (b) Packets drops for each layer in scenario 2
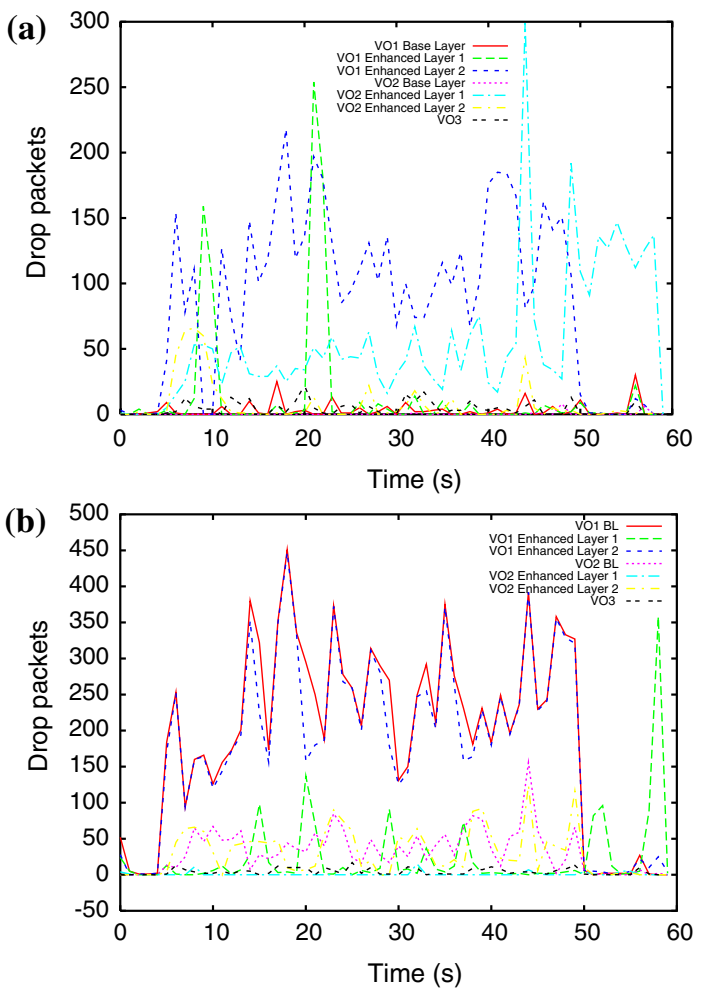

end-user. The proposed quality adaptive streaming mechanism is based on the construction of overlay networks which are constructed based-on active monitoring which is performed by tracking "RTT', between the received and potential sender 


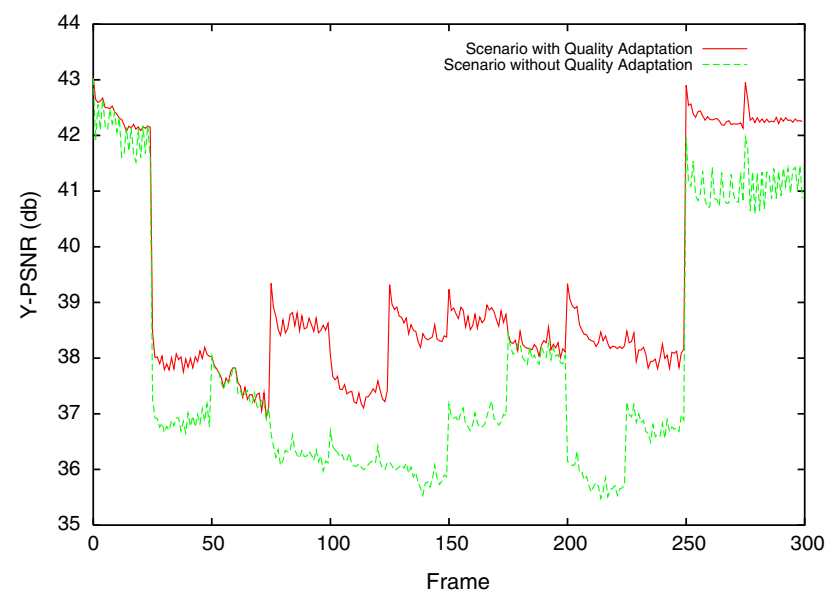

Fig. 12 PSNR measurement for both scenarios

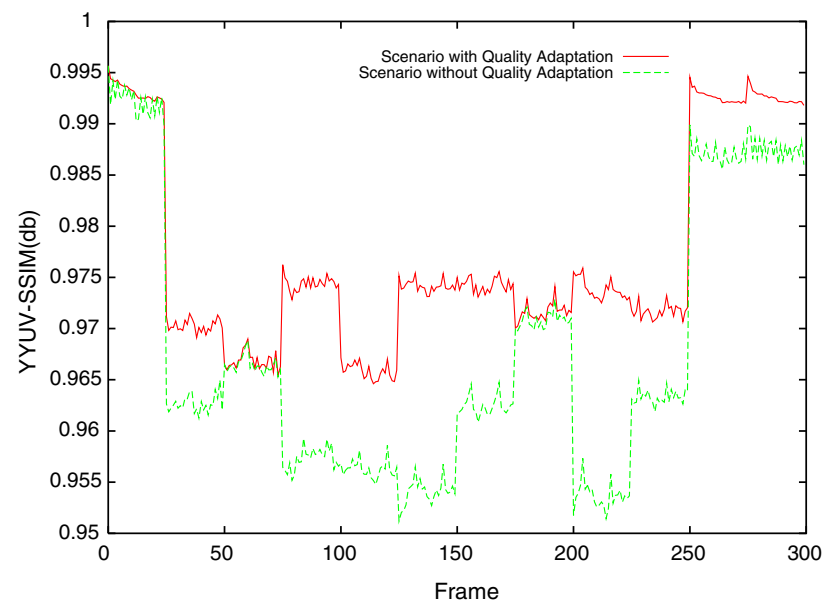

Fig. 13 SSIM measurement for both scenarios

peers. The overall purpose of this study was to ensure the delivery of most important video layers, i.e. base layers of each object. Our simulation results show that using the proposed mechanism a noticeable improvement of received throughput and lower packet loss in the network which results in a better overall quality of service. A smallest amount of packet drops are observed for the most important objects. We have witnessed that dynamic behavior of sender peers doest not affect much to the received quality of video because of our efficient peer switching mechanism. For the future prospective, we are investigating the issues regarding multi path routing, many to many communications, where many receiver peers intend to receive media packets from multiple sender peers. 

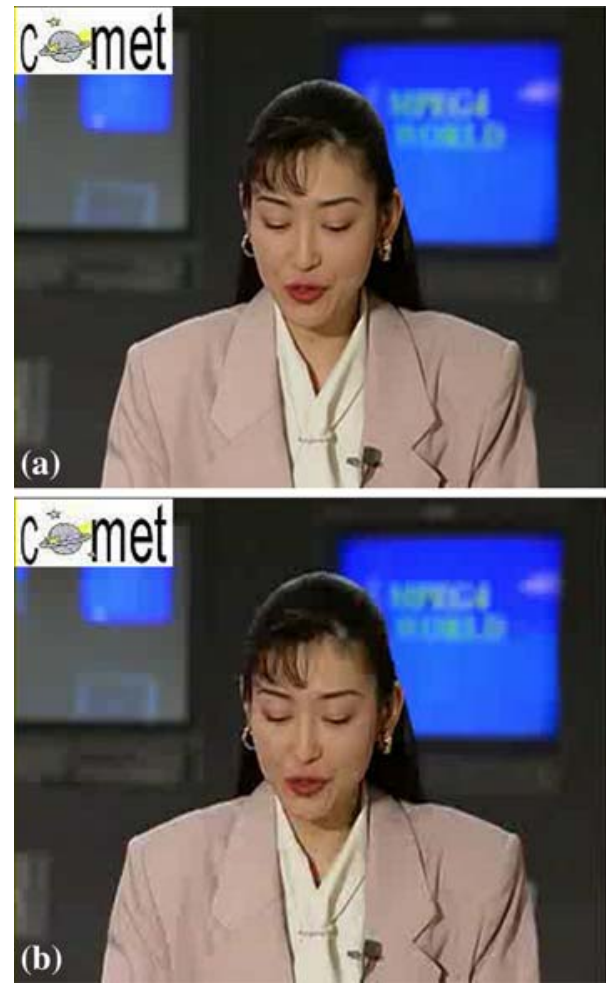

Fig. 14 (a) Snapshot of received Video in scenario 1; (b) Snapshot of received video in scenario 2

Acknowledgements The authors would like to thank the anonymous reviewers for their helpful and constructive comments on the submitted manuscript, which improved the presentation and the quality of this paper significantly.

\section{References}

1. Amon, P., Pandel, J.: Evaluation of adaptive and reliable video transmission technologies. Packet Video'03, France (2003)

2. Sun, X., Li, S., Wu, F., Shen, G., Gao, W.: Efficient and flexible drift-free video bitstream switching at predictive frames. ICME (2002)

3. Sun, X., Wu, F., Li, S., Gao, W., Zhang, Y.Q.: Seamless switching of scalable video bitstreams for efficient streaming. In: IEEE International Symposium on Circuits and Systems, ISCAS 2002, Scottsdale, Arizona, USA, 26-29 May (2002)

4. Chou, Y.K., Jian, L.C., WenLin, C.: MPEG-4 video streaming with drift-compensated bit-stream switching. In: Proc. IEEE Pacific-Rim Conf. Multimedia, 847-855, Dec (2002)

5. Real Network: RealSystem G2: management and control of streaming media over corporate networks RealVideo. Available at http://docs.real.com/docs/devzone/g2ctrlandmgmt.pdf, Mar (1999)

6. Wee, S., Apostolopoulos, J., Feamster, N.: Field-to-Frame transcoding with temporal and spatial downsampling. In: IEEE International Conference on Image Processing, Oct (1999)

7. Amir, E., McCanne, S., Zhang, H.: An application level video gateway. In: Proc. ACM Multimedia (1995) 
8. Amir, E., McCanne, S., Katz, R.: An active service framework and its application to real-time multimedia transcoding. In: SIGCOMM, symposium on communications architectures and protocols, Sept (1998)

9. Wang, B., Sen, S., Adler, M., Towsley, D.: Proxybased distribution of streaming video over unicast/ multicast connections. Technical Report UMASS TR-2001-05, University of Massachusetts, Amherst (2001)

10. Chan, S.-H.G., Tobagi, F.A.: Caching schemes for distributed video services. In: Proceedings of the IEEE International Conference on Communications (IEEE ICC), Vancouver, Canada, June (1999)

11. Chuang, J.: Distributed network storage service with quality-of-service guarantees. J. Network Comput. Appl. 23(3), 163-185 (2000)

12. Kangasharju, J., Hartanto, F., Reisslein, M., Ross, K.W.: Distributing layered encoded video through caches. In: Proceedings of the Conference on Computer Communications (IEEE Infocom), Anchorage, Alaska, April (2001)

13. Zhang, Z.L., Wang, Y., Du, D.H.C., Su, D.: Video staging: a proxy-server-based approach to end-toend video delivery over widearea networks. IEEE/ACM Trans. Network. 8(4), 429-442 (2000)

14. Rejaie, R., Kangasharju, J.: Mocha: a quality adaptive multimedia proxy cache for internet streaming. In: Proceedings of NOSSDAV'01, June (2001)

15. Lam, S.S., Chow, S., Yau, D.K.Y.: A lossless smoothing algorithm for compressed video. IEEE/ ACM Trans. Network. 4(5), 697-708 (1996)

16. Salehi, J.D., Zhang, Z.-L., Kurose, J.F., Towsley, D.: Supporting stored video: reducing rate variability and end-to-end resource requirements through optimal smoothing. IEEE/ACM Trans. Network. 6, 397-410 (1998)

17. Ng, J.K.-Y., Shibin, S.: A video smoothing algorithm for transmitting MPEG video over limited bandwidth. In: Proc. of the 4th International Workshop on Real-Time Computing Systems and Applications (RTCSA '97)

18. Mansour, Y., Patt-Shamir, B., Lapid, O.: Optimal smoothing schedules for real-time streams. In: ACM Principles of Distributed Computing, Portland, OR (2000)

19. Anastasiadis, S.V., Sevcik, K.C., Stumm, M.: Server-based smoothing of variable bit-rate streams. In: Proceedings of the 9th ACM Multimedia Conference, 147-158, Ottawa, October (2001)

20. Gkantsidis, C., Rodriguez, P.: Network coding for large scale content distribution. IEEE/INFOCOM'05, Miami (2005)

21. Rejaie, R., Ortega, A.: PALS: peer-to-peer adaptive layered streaming. In: Proceedings of the International Workshop on Network and Operating Systems Support for Digital Audio and Video, Monterey, California, June (2003)

22. Hefeeda, M., Habib, A., Botev, B., Xu, D., Bharat B.: Promise: peer-to-peer media streaming using collectcast. ACM MM'03, Berkeley, CA (2003)

23. Nguyen, T., Zakhor A.: Multiple sender distributed video streaming. IEEE Trans. Multimedia 6(2), 315-326 (2004)

24. Yeo, C.K., Lee, B.S., Er, M.H.: A framework for multicast video streaming over IP networks. J. Network Comput. Appl. 26, 273-289 (2003)

25. Loeser, C., Ditze, M., Altenbernd, P.: Architecture of an intelligent quality-of-service aware peer-topeer multimedia network. In: Proc. of the 7th World of Multiconference on Systemics, Cybernetics and Informatics, July (2003)

26. Loeser, C., Ditze, M., Altenbernd, P., Ramming, F.: GRUSEL—a self optimizing bandwidth aware video on demand P2P application. In: Proceedings of the International Conference on Autonomic Computing (ICAC'04), 330-331

27. Guo, Y., Suh, K., Kurose, J., Don, T.: P2Cast: peer-to-peer patching scheme for VoD service" In: Proceedings of the 12th World Wide Web Conference (WWW-03), Budapest, Hungary, May (2003)

28. Tran, D.A., Hua, K.A., Do, T.T.: ZIGZAG: an efficient peer-to-peer scheme for media streaming. In: Proceedings of IEEE INFOCOM 2003, San Francisco, CA, March (2003)

29. Tran, D.A., Hua, K.A., Do, T.T.: A peer-to-peer architecture for media streaming. IEEE J. Selected Areas Commun. 22(1), 121-133 (2004)

30. Padmanabhan, V.N., Wang, H.J., Chou, P.A., Sripanidkulchai, K.: Distributing streaming media content using cooperative networking. ACM NOSSDAV, Miami Beach, FL, USA (2002)

31. Padmanabhan, V.N., Wang, H.J., Chou, P.A.: Resilient peer-to-peer streaming. IEEE ICNP, Atlanta, GA, USA (2003) 
32. Ahmed, T., Nafaa, A., Mehaoua, A.: An object-based MPEG-4 multimedia content classification model for IP QoS differentiation. In: Proceedings of the 8th IEEE Symposium on computers and communications ISCC'03, 1091-1096, July (2003)

33. Stoica, I., Morris, R., Karger, D., Kaashoek, M. F., Balakrishnan H.: Chord: a scalable peer-to-peer lookup service for internet applications. ACM SIGCOMM (2001)

34. Jacobson, V.: Congestion avoidance and control. ACM SIGCOMM (1988)

35. Fitzek, F.H.P., Reisslein M.: MPEG-4 and H.263 video traces for network performance evaluation. IEEE Network 5(6), 40-54, (2001)

\section{Author Biographies}

Toufik Ahmed received his computer Engineer degree (Honors) from I.N.I (Institut National d'Informatique), Algiers, Algeria in 1999 and Master's and Ph.D. degrees in computer science from University of Versailles in 2000 and 2003, respectively. He is currently an Associate Professor at ENSEIRB School of engineers/University of Bordeaux I, Bordeaux, France. His main research activities concern QoS for multimedia wired and wireless networks, cross-layer optimization, and end-to-end QoS signaling protocols. His work on quality of service and video delivering has led to many publications in major journals and conferences.

Mubashar Mushtaq is currently pursuing his PhD degree from CNRS LaBRI Lab-University of Bordeaux 1. He has received his MSc and MS degrees in Computer Science from Quaide-i-Azam University, Islamabad, Pakistan and University of Bordeaux 1, France in 2002 and 2004, respectively. His research interests include Multimedia Streaming over next generation networks, QoS, mobile IP-TV, and multimedia caching. 